\title{
Structural and Compositional Transitions at the Atomic Scale Across Interfaces, Faults and Phase Separations in Complex Oxides
}

\author{
R.E.A. Williams*, M. Dixit*, R. Mishra, A. Genc**, R. Srinivasan***, G.B. \\ Viswanathan****, D.W. McComb ${ }^{* * * *}$, J. Ringnalda******, C. Dwyer*******, W. \\ Windl*, H.L. Fraser* \\ *Center for the Accelerated Maturation of Materials and Department of Materials \\ Science and Engineering, The Ohio State University, Columbus, OH 43210, U.S.A. \\ **Intel Inc., Hillsboro, OR 97124 \\ ***ExxonMobil Research and Engineering Company, Annandale NJ 08801 \\ **** UES Inc., Dayton, OH \\ *****Department of Materials, Imperial College London, London, SW7 2AZ, United \\ Kingdom \\ ******FEI Company, 5350 NE Dawson Creek Drive, Hillsboro, OR 97124, USA
}

The advent of aberration-corrected microscopy has enabled the coupling of structural information with spatially resolved compositional information [1-3]. Several studies have shown the ability to study composition at the atomic scale through core-loss spectroscopy. However, it is also important to consider the delocalization of the EELS signal [3-5], while attempting quantitative information on atomic-scale composition.

At the outset, this study attempts to highlight some of the key factors controlling elemental quantification at the atomic scale. Through a detailed study using strontium titanate as a model system, the importance of the apparent delocalization of the EELS signal is highlighted. For example, in an EELS line profile along $\mathrm{Sr}-\mathrm{O}-\mathrm{Sr}$ atomic columns in [001] oriented $\mathrm{SrTiO}_{3}$, the modulation of the Ti-L signal and its maximization on $\mathrm{O}$ columns can be observed (FIG 1a). EELS line profiles along the Ti/O-O-Ti/O atomic columns, as shown in FIG $1 \mathrm{~b}$, demonstrate the modulation of the $\mathrm{O}-\mathrm{K}$ signal, and its maximization on the Ti/O columns, even though the linear density of $\mathrm{O}$ atoms is the same along both the $\mathrm{Ti} / \mathrm{O}$ and pure $\mathrm{O}$ columns in [001]-oriented $\mathrm{SrTiO}_{3}$. Possible factors affecting such a behavior will be discussed.

As a related and continuing study, the nature of faults and interfaces in off-stoichiometric $\mathrm{SrTiO}_{3}$ films (grown on stoichiometric STO substrates) is investigated. While the direct interpretation of EELS signals at the atomic resolution can be complicated, the ability to directly interpret HAADF-STEM images at the atomic scale (i.e. HAADF-HRSTEM) enables us to identify the nature of planar faults in Sr-rich compositions (see Figure 2a). Based upon the experimental observations, a simple mathematical model is proposed to explain the nature of these planar faults in Sr-rich compositions, similar to the Ruddlesden-Popper (RP) faults observed in other studies [references: see our draft paper on Sr-rich STO]). On the other hand, deviations to the Sr-deficient compositions appear to trigger a rather interesting phase separation mechanism (see Figure 2b). These experimental observations are supplemented with the results from atomistic and DFT calculations aimed at understanding the stability and energetics of various defect 
structures in off-stoichiometric STO compositions [reference: our work as unpublished research].

Support for this work, provided by the National Science Foundation for the Materials Research Science and Engineering Center (MRSEC) under contract number DMR0820414 is gratefully acknowledged.

References

[1] D.A. Muller, et al., Nature 366, (1993) 725

[2] E.C. Cosgriff, et al., Ultramicroscopy 102, (2005) 317

[3] L.J. Allen, et al., Phys. Rev. Lett. 91, (2003)105503-1

[4] C. Dwyer, J. Etheridge, Ultramicroscopy 96, 343 (2003)

[5] H.L. Fraser, et al., unpublished research
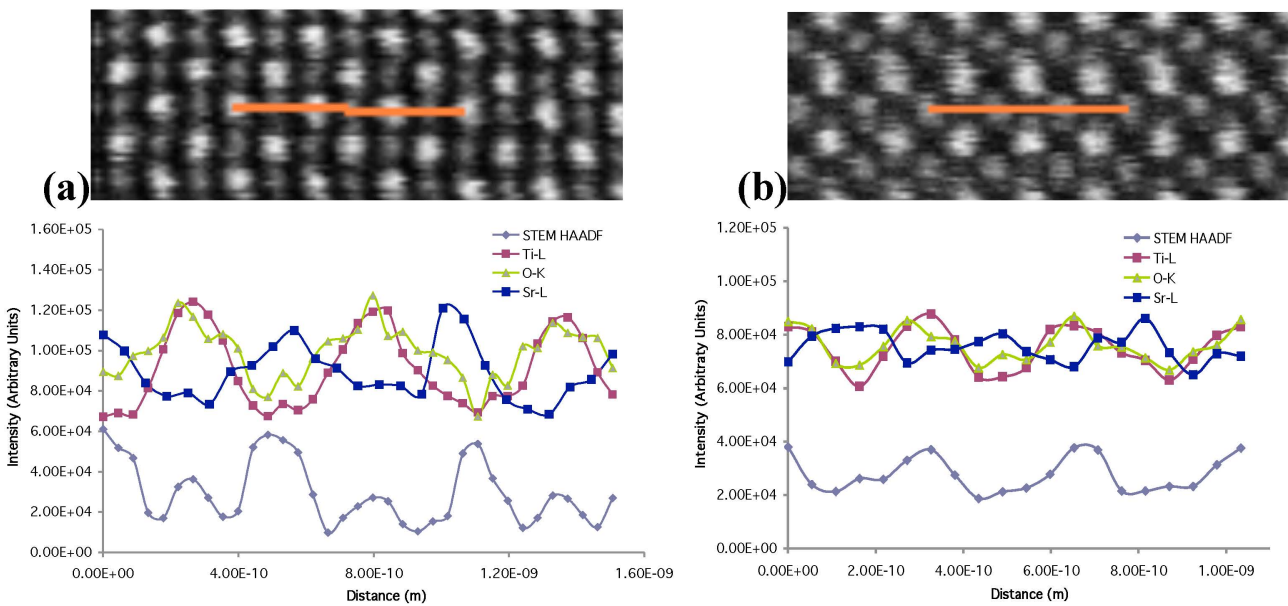

FIG 1: EELS signals for line profiles across (a) $\mathrm{Sr}-\mathrm{Ti} / \mathrm{O}-\mathrm{Sr}$ columns, (b) $\mathrm{Ti} / \mathrm{O}-\mathrm{O}-\mathrm{Ti} / \mathrm{O}$ columns. Corresponding HAADF-HRSTEM images for each case are also shown

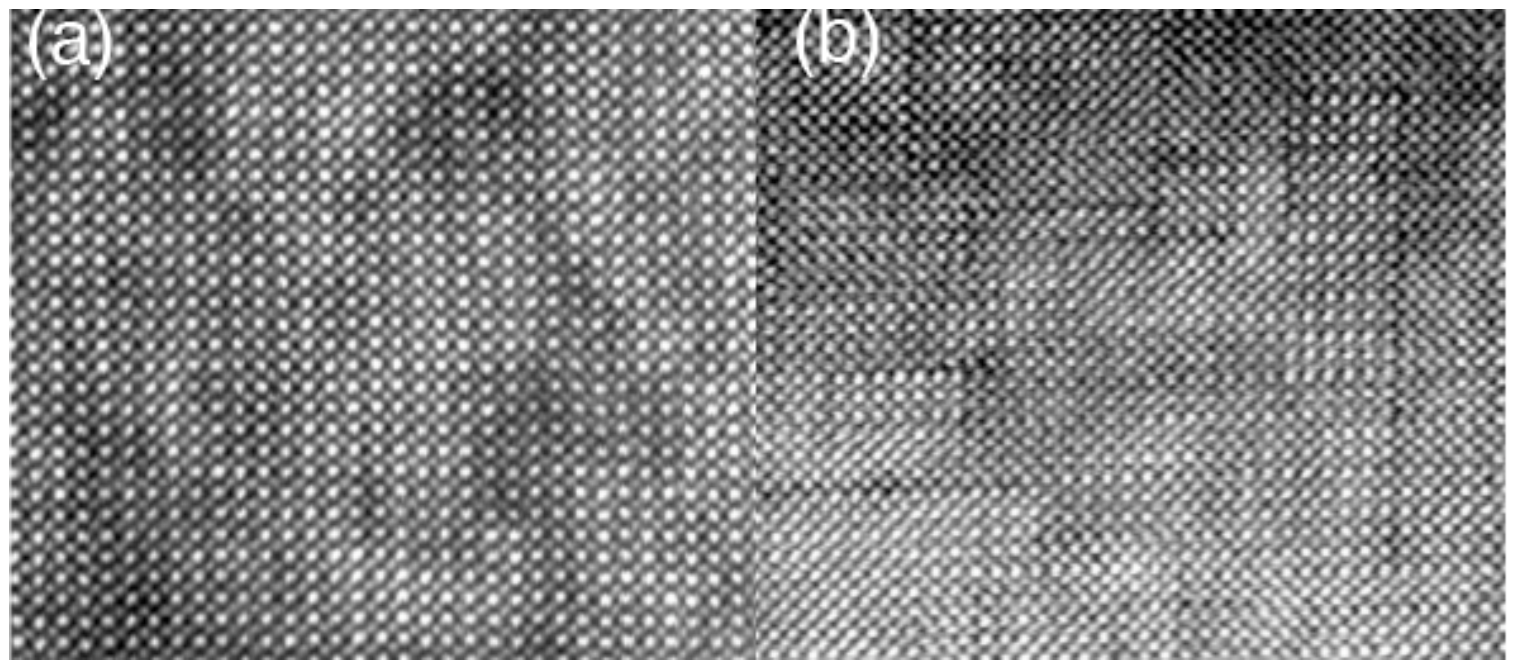

FIG 2: Atomic Resolution HAADF-STEM images of (a) Sr deficient STO, (b) Sr rich STO 